\title{
Considering Too Few Alternatives: The Mental Model Theory of Extensional Reasoning
}

\author{
Thierry Chevalley, Walter Schaeken \\ KU Leuven Laboratory of Experimental Psychology \\ University of Leuven, \\ Tiensestraat 102 \\ 3000 Leuven, Belgium \\ Thierry.Chevalley@ppw.kuleuven.be \\ Walter.Schaeken@ppw.kuleuven.be \\ Short Title: \\ Considering Too Few Alternatives
}

Corresponding author:

Thierry Chevalley

KU Leuven Laboratory of Experimental Psychology

University of Leuven, box 037111

Tiensestraat 102

3000 Leuven, Belgium

Tel. +3216325968

Fax. +3216326099

Thierry.Chevalley@ppw.kuleuven.be

\section{Acknowledgements}

We would like to thank FWO Flemish Research Fund. This work was supported by the Research Council of the University of Leuven, project OT/11/030. 


\begin{abstract}
When solving a simple probabilistic problem, people tend to build an incomplete mental representation. We observe this pattern in responses to probabilistic problems over a set of premises using the conjunction, disjunction and conditional propositional connectives. The Mental Model Theory of Extensional Reasoning explains this bias towards underestimating the number of possibilities: In reckoning with different interpretations of the premises (logical rules, mental model theoretical, and specific to conditional premises, conjunction and biconditional interpretation) the Mental Model Theory accounts for the majority of observations. Different interpretations of a premise result in a build-up of mental models that are often incomplete. These mental models are processed using either an extensional strategy relying on proportions amongst models, or a conflict monitoring strategy. The consequence of considering too few possibilities is an erroneous probability estimate akin to that faced by decision makers who fail to generate and consider all alternatives, a characteristic of bounded rationality. We compare our results to the results published Johnson-Laird, Legrenzi, Girotto, Legrenzi, and Caverni (1999), and observe lower performance levels than in the original article.
\end{abstract}

Keywords: probability, mental models, elicitation of estimates, extensional reasoning. 
Out of 57 participants in a probability estimating experiment, eight respondents are classified by the experimenter as one-model thinkers. What answer pattern would correspond to such a classification? When asked to work with the conditional If there is a yellow card in the box, then there is a black card, they respond with the conjunctive interpretation of the conditional (i.e. When there is a yellow card, there is also a black card) and consistently consider only one possibility. This affects their performance in other tasks: When the premise includes an exclusive disjunction In a box, you will get either a red card or a green card, but not both, they make correct probability estimates $66.7 \%$ of the time. In case of an inclusive disjunction, a red card or a green card, or both premise that offers three possibilities, their score drops to $37.5 \%$, at chance level and below the average observed for all respondents.

Is this an instance of bounded rationality when facing a complex problem? Do these participants overlook some possibilities which an otherwise rational agent ought to consider, thus failing to evaluate all alternatives before making a choice (Simon, 1957)? A person reasoning about a specific problem has to construct a simplified model of reality in order to deal with it. This is based on the principle of bounded rationality: "the capacity of the human mind for formulating and solving complex problems is very small compared with the size of the problems whose solution is required for objectively rational behaviour in the real world - or even for a reasonable approximation to such objective rationality" (Simon, 1957, p. 198). It is of interest to know how novices and experts handle probabilistic problems because, in situations of uncertainty and risk, probabilistic reasoning is needed to find the most likely possibility. Amongst the constraints described by Simon (1957) are physiological and psychological limits on the organism and on computational capacity. This overlooking of possibilities contrasts with the purely rational view that decision makers should choose the best alternative after generating all possible alternatives. We observe when testing people's reasoning competence in estimating probabilities that they omit mental representations would be required to capture the problem adequately. Their behaviour is one of the psychological limitations placed on rational problem solving.

This paper outlines two experiments conducted to investigate extensional reasoning with small integer numbers to estimate probabilities. The theoretical framework is the Mental Model Theory, which explains the build-up of mental representations. To evaluate the Mental Model Theory of Extensional Reasoning (Johnson-Laird, Legrenzi, Girotto, Legrenzi, \& Caverni, 1999), we inquire how people apply extensional reasoning strategies with mental models to estimate probabilities. Reasoning about probabilities with an extensional approach requires that the different possible ways in which an event could occur be considered (Johnson-Laird et al., 1999). This raises the issue of what is mentally represented and how it is processed. First, we consider the nature and number of mental representations that a reasoner builds to respond to a specific situation; second, we look at which reasoning strategy one may use to process the mental models. In 1999, Johnson-Laird et al. presented a new application of mental models to probabilistic reasoning, the 'Naïve Probability' Theory. This theory postulates that reasoners can infer the probabilities of events in an extensional way by constructing mental models of the various possibilities in which the event can occur. The goal of this theory is to explain how people who are unfamiliar with probability calculus reason with probabilities when facing a 
situation with a small number of alternatives.

The Mental Model Theory suggests that individuals represent as little information as possible (Johnson-Laird, Byrne, \& Schaeken, 1992): Based on this parsimony of representing information and with the distinction between initial and explicit mental models, the Mental Model Theory explains why individuals tend to build too few mental models. Building on the initial representation, reasoners may flesh out certain mental models depending on the information provided and drop other models once they are deemed to be of no further utility. In particular, it is assumed that only those mental models are constructed which represent what is true in a true possibility, as stated in the truth principle by Johnson-Laird et al. (1999).

Our approach to investigate reasoning with small numbers and simple probabilities is to ask questions that use simple combinations of objects, one of each kind, and present these problems to the participant who evaluates them against a premise: An example is 'There is a box in which there is a green card, or a red card, or both' followed by the request to estimate the probability of finding inside the box 'a green card without a red card'.

Our purpose is to establish the scope of application of the Mental Model Theory of Extensional Reasoning and to measure the proportion at which people underestimate the number of mental representations, which is a characteristic of bounded rationality. The questions we pose are:

What fraction of probability estimates can extensional reasoning explain? When is the hypothesis of a parsimonious build-up of representations (Johnson-Laird \& Byrne, 1991) applicable? For which response patterns can the Mental Model Theory offer an explanation, taking different interpretations (material implication, conjunction, biconditional and mental model theoretical) of a (conditional) premise into account? Other questions we address in Experiment 1 are: When is a reasoning strategy based on performance monitoring and conflict detection an alternative to extensional reasoning? What are the relative difficulties of the conjunction, disjunction and conditional propositional connectives? Are there conjunction fallacies? Does the method of eliciting probability estimates by varying the entry mode of responses and mitigating guessed responses affect results?

\section{Mental Representation}

People build mental representations to describe a given situation: These representations refer to the objects that have just been mentioned, e.g. a green card, a blue card. Mental models are representations of the possible combinations of these objects. The model theory further posits that people retain only the positive propositions: The truth principle states that people construct sets of mental models in which each model represents what is true in a true possibility. Situations that cannot occur coincide with what is false and their propositions are dropped (Johnson-Laird et al., 1999). The analysis of the conditional If there is a green card, then there is a blue card using the Mental Model Theory illustrates the parsimonious build-up of mental representations: The first model is an explicit model of the possibility in which the antecedent and consequent of the conditional are true. Other logically true possibilities are those in which the antecedent of the conditional is false: These possibilities are not normally 
represented explicitly. Instead, "these possibilities are represented in a single implicit model, a model that has no explicit content and that we symbolise with an ellipsis (three dots)" (JohnsonLaird et al., 1999, p. 67). The initial representation of the conditional has two mental models, one explicit and the other wholly implicit:

$$
\text { green card blue card }
$$

As in Table 1 we follow the notational convention of Johnson-Laird et al. (1992) and Johnson-Laird and Byrne (1991) where separate models are listed on separate lines.

(Insert Table 1 about here)

By default each model represents an equiprobable alternative (Johnson-Laird et al., 1999). This

Table 1

Mental Models for Propositional Connectives

\begin{tabular}{|c|c|c|c|c|}
\hline Premise & \multicolumn{2}{|c|}{ Initial Mental Models } & \multicolumn{2}{|c|}{ Fully Explicit Models } \\
\hline$A$ and $B$ & A & B & A & B \\
\hline \multirow[t]{2}{*}{ A or else $B$} & $\mathrm{~A}$ & & A & $\neg \mathrm{B}$ \\
\hline & & B & $\neg \mathrm{A}$ & B \\
\hline \multirow[t]{3}{*}{ A or B or both } & A & B & A & B \\
\hline & A & & A & $\neg \mathrm{B}$ \\
\hline & & B & $\neg \mathrm{A}$ & B \\
\hline \multirow[t]{3}{*}{ If $\mathrm{A}$, then $\mathrm{B}$} & A & B & A & B \\
\hline & & & $\neg \mathrm{A}$ & B \\
\hline & & & $\neg \mathrm{A}$ & $\neg \mathrm{B}$ \\
\hline \multirow[t]{2}{*}{ If, and only if $A$, then $B$} & A & B & A & B \\
\hline & & & $\neg \mathrm{A}$ & $\neg \mathrm{B}$ \\
\hline
\end{tabular}

Note. The $\neg$ indicates a negation. ... stands for an implicit model.

assumption is also described as the equiprobability principle in Johnson-Laird et al. (1999). The term equiprobability refers to situations where all models are equal in the probability of occurrence, as derived from the equal number of possibilities of their occurrence. For example, tossing a fair coin once has two possible outcomes, head or tail, each with an equal possibility of occurrence. The mental models constructed to represent the possible outcomes of a fair coin toss are Head and Tail, each alternative having an equal probability. In this case of two models, the probability is equal to one-half. Based on the truth and equiprobability principles, the theory predicts several phenomena of reasoning about absolute probabilities, including typical biases.

\section{Reasoning Strategy}

Given the representation that is built-up, the processing of mental models that make up the representation can be described using different reasoning strategies. We consider the extensional 
strategy of comparing the proportion of mental models, and the detection of contradictions in the evidence provided.

The extensional way of reasoning about probabilities implies that individuals "construct mental models of what is true in the various possibilities. Each model represents an equiprobable alternative unless individuals have beliefs to the contrary, in which case some models will have higher probabilities than others. The probability of an event depends on the proportion of models in which it occurs" (Johnson-Laird et al., 1999, p. 62). The proportionality principle described by Johnson-Laird et al. (1999, p. 68) states that "granted equiprobability, the probability of an event, $A$, depends on the proportion of models in which the event occurs; that is, $p(A)=\frac{n_{A}}{n}$, where $n_{A}$ is the number of models containing $A$, and $n$ is the number of models" . Alternatively, in case the information processed contains a contradiction, the reasoner can opt for a strategy of detecting contradictions. This is best illustrated by the conditional premise If $A$, then $B$ combined with the contradicting problem $p(A$ and $\neg B)$ : Johnson-Laird et al. (1999) reported that $100 \%$ of their participants correctly estimated the probability at $0 \%$.

Situations where the information contains a contradiction are the subject of separate investigations in cognitive neuropsychology. Neural correlates of cognitive control and conflict monitoring are well characterised: Conflicting situations are special in terms of the pattern of brain resources they recruit (Ridderinkhof, van den Wildenberg, Segalowitz, \& Carter, 2004). To check on such an effect, we probe the difference in performance between questions using contradicting information and questions that are free of apparent contradictions.

\section{Interpretation of Conditionals}

People can interpret a conditional statement If $A$, then $B$ in different ways, as reported in conditional inference tasks (Evans, Handley, Neilens, \& Over, 2007; Oberauer, Geiger, Fischer, \& Weidenfeld, 2007). With our experimental task, these interpretations lead to different responses that fall into separate categories of forecasted answers. Several theories can explain the interpretation of a conditional: formal rules of logic, the theoretical build-up of mental models, and suppositional theories based on Ramsey's test with the defective truth table (Byrne \& JohnsonLaird, 2009). Thompson (2000, p. 212) explains that "both variability and consistency in performance arise from the action of the interpretive component, which completely determines the number and nature of the inferential procedures that are recruited". Hence, we consider four interpretations of the conditional: conjunction, biconditional, mental model theoretical buildup of initial models, and the material implication derived from formal logic. These correspond to the interpretations described at different developmental stages (three successive interpretational levels: conjunctive, biconditional, and then conditional) by Barrouillet, Gauffroy, and Lecas (2008) in the most recent revised version of the Mental Model Theory for conditionals. The build-up of mental models can readily explain the biconditional and conjunction interpretations of If $A$, then $B$, as well as its interpretation according to formal rules of logic (Byrne \& Johnson-Laird, 2009; Evans et al., 2007; Oberauer et al., 2007).

Another interpretation observed in conditional inference tasks is the defective truth table, which builds on the idea that "Subjects assume implicitly that a conditional statement has, not two truth values, but three: true, false, irrelevant" (Wason, 1966, p. 146). In conditional in- 
ference experiments involving truth table tasks, the defective truth table pattern that includes the judgement that propositions with a negative antecedent $\neg A$ are irrelevant, is a common answer (Evans, Handley, Neilens, \& Over, 2008). These interpretations bring forth the different forecasted response patterns listed in Table 2, based on models described in Table 1: The forecast for the material implication interpretation is derived from the fully explicit models for If $A$, then $B$, the Mental Model Theory forecast from the initial mental models for If $A$, then $B$, and the conjunctive interpretation from the mental models for the conjunction $A$ and $B$. With the premises and problems used in our experiment, the biconditional interpretation If, and only if $A$, then $B$ listed in Table 1 yields the same forecasted values as the mental model theoretical interpretation of the conditional If $A$, then $B$. We do not include the defective truth table interpretation in our analysis because we could not make a reliable prediction for a problem contradicting the premise.

We shall consider logically correct responses, biased responses as predicted by the Mental Model Theory and by the biconditional and conjunctive interpretation of the premise, and other incorrect answers. A detailed look at the distribution of responses question by question offers further indication of the difficulty of premises; a wide distribution of responses along the entire scale of possible probabilities is indicative of a wide variety of interpretations chosen by the different respondents. On the other hand, one cluster (or a few clusters) of responses around a particular value indicates a consensus amongst respondents about the premise and problem presented. Individual answers inform us about the number of mental models a participant used in response to a situation described in the premises; this estimate can then be compared against the number of mental models implied by the logical norm, and a trend for the typical bias may be found. This detailed approach is better suited to this experiment and more informative than merely computing the average of responses given, particularly when the responses are not clustered around one value, but spread over the entire available scale.

\section{Conjunction Fallacy}

Another way to inquire into people's rationality is to look for the conjunction fallacy, a logical fallacy whereby the likelihood of a conjunction of two events occurring is erroneously estimated to be higher than the probabilities of its conjuncts. The Mental Model Theory predicts a conjunction fallacy when a reasoner leaves out certain models of the representation. For instance with the conditional If $A$, then $B$, "the fallacy would occur if a participant forgot the implicit model in assessing $p(A$ and $B)$, which would yield a probability of $100 \%$, but did not forget the implicit model in assessing $p(A)$, which would yield a probability of 50\%" (Johnson-Laird et al., 1999, p. 71). To avoid the conjunction fallacy, one can follow the conjunction rule for the probability $\mathrm{p}(\mathrm{A}$ and $\mathrm{B}): p(A$ and $B) \leq p(A), p(A$ and $B) \leq p(B)$. The abstract formulation of questions used in our experiment is belief neutral and precludes the use of representativeness and availability heuristics. Respondents solve each problem individually and do not check their answers for consistency with the conjunction rule (Tversky \& Kahneman, 1983). This instance of bounded rationality in extensional reasoning with abstract questions is explained by underestimating the number of mental models. 


\section{A discerning way of eliciting probability estimates}

Because respondents may find some questions difficult and guess a response, and because a third of the problems posed in our two experiments yield a 50\% value predicted by the Mental Model Theory, we need to reduce any expression of maximum uncertainty or pure guess as a $50 \%$ response that would otherwise over-emphasize the middle of the probability scale. In Experiment 1, we use premises mentioning two objects that can induce a binary case partition prior to any further processing of the information; this binary case partition constrains the 'ignorance-prior' probability to 50\% (Fox \& Rottenstreich, 2003, p. 195).

To reduce the potential impact of 50\% guessed responses and optimise the elicitation of probabilities, we take two methodological steps: (1) Use two response entry modes, a graphical entry mode with a scale printed on paper provided for respondents to mark their response, and a numerical entry mode with an underlined field for respondents to write down their answer. (2) Place an additional entry field next to the response scale (or next to the numerical response entry respectively) labelled 'I do not know an answer'; we shall refer to these responses as no-clue answers. This no-clue field is equivalent to the 'absolutely no idea' field used by Fischhoff and Bruine de Bruin (1999, p. 151).

We strive to insure the reliability of the data and to quantify any effect of the entry mode on the accuracy and spread of answers (usage of the scale), as well as to reduce biases related to respondents expressing their uncertainty about the question or guessing (Fischhoff \& Bruine de Bruin, 1999). Studies by Carey and White (1991) reported differences between both response modes when measuring the accuracy of extrapolating a trend. Specific to the elicitation of probabilities, the study by Fischhoff and Bruine de Bruin (1999) reports a significant drop in 50\% responses when responding on a graphical scale, compared to a blank numerical entry field; hence we also use the graphical entry mode with a paper questionnaire for the ease of marking a point on the scale with a pencil. Studies on the use of computer-based testing have traditionally shown better comprehension with paper (Christensen, 2013) and more workload with a computer (see Noyes and Garland, 2008, for a review). Differences in reading comprehension and scores between computer and paper have been decreasing over the years, but equivalence in computer- and paper-based tasks has not been achieved yet.

The no-clue field and the two entry modes have been introduced to avoid $50 \%$ guesses. By separating responses where a respondent might indicate $50 \%$ because they do not know the answer, we prefer to avoid accruing guessed responses along the Mental Model Theory's predicted value. We shall keep track of the mitigation of guessed responses by monitoring the number of no-clue answers, the incidence of 50\% answers, the accuracy of responses and usage of scale, in Experiment 1 and in the General Discussion. 


\section{Experiment 1}

\section{Introduction and Experimental Design}

The experimental task is a set of problems built around logical premises that each describe a specific situation. The premises use the conjunction, disjunctions and conditional connectives, equivalent to the task used by Johnson-Laird et al. (1999). Combining each premise with five problem items, we ask participants to estimate the likelihood of a given problem occurring relative to the rule specified in the premise. Example of a typical premise and problem combination: Premise: There is a box in which there is a white cup, or a blue cup, or both.

Problem: Please estimate the probability in percentages (from 0\% [never true] to $100 \%$ [always true]) that the following statement is true: In the box there is at least a white cup. Probability: ... \%

The four premises used in Experiment 1 are listed in Table 2, together with the probability estimating problems. Table 2 presents the norms derived from the mental models listed in Table 1. The predicted value is the fraction of the number of occurrences of the problem item in the mental representation, divided by the extension of all models built to represent the premise.

(Insert Table 2 about here)

Table 2

Predictions for the probability estimates by premise and problem item

\begin{tabular}{|c|c|c|c|c|c|}
\hline Premise & \multicolumn{5}{|c|}{ Predicted response by problem item } \\
\hline Conjunction & $\mathrm{p}(\mathrm{A})$ & $\mathrm{p}(\mathrm{A} \wedge \mathrm{B})$ & $\mathrm{p}(\mathrm{A} \wedge \neg \mathrm{B})$ & $\mathrm{p}(\neg \mathrm{A} \wedge \mathrm{B})$ & $\mathrm{p}(\neg \mathrm{A} \wedge \neg \mathrm{B})$ \\
\hline$A$ and $B$ & $100.0 \%$ & $100.0 \%$ & $0.0 \%$ & $0.0 \%$ & $0.0 \%$ \\
\hline Disjunctions & $\mathrm{p}(\mathrm{A})$ & $\mathrm{p}(\mathrm{A} \wedge \mathrm{B})$ & $\mathrm{p}(\mathrm{A} \wedge \neg \mathrm{B})$ & $\mathrm{p}(\neg \mathrm{A} \wedge \mathrm{B})$ & $\mathrm{p}(\neg \mathrm{A} \wedge \neg \mathrm{B})$ \\
\hline A xor B, but not both & $50.0 \%$ & $0.0 \%$ & $50.0 \%$ & $50.0 \%$ & $0.0 \%$ \\
\hline$A$ or $B$ & $66.7 \%$ & $33.3 \%$ & $33.3 \%$ & $33.3 \%$ & $0.0 \%$ \\
\hline $\begin{array}{l}\text { Conditional If } A \text {, then } B \\
\text { Interpretations: }\end{array}$ & $\mathrm{p}(\mathrm{A})$ & $\mathrm{p}(\mathrm{A} \wedge \mathrm{B})$ & $\mathrm{p}(\mathrm{A} \wedge \neg \mathrm{B})$ & $\mathrm{p}(\neg \mathrm{A} \wedge \mathrm{B})$ & $\mathrm{p}(\neg \mathrm{A} \wedge \neg \mathrm{B})$ \\
\hline - Mental model theoretical & $50.0 \%$ & $50.0 \%$ & $0.0 \%$ & $0.0 \%$ & $50.0 \%$ \\
\hline - Logical fully explicit models & $33.3 \%$ & $33.3 \%$ & $0.0 \%$ & $33.3 \%$ & $33.3 \%$ \\
\hline - Biconditional & $50.0 \%$ & $50.0 \%$ & $0.0 \%$ & $0.0 \%$ & $50.0 \%$ \\
\hline - Conjunction & $100.0 \%$ & $100.0 \%$ & $0.0 \%$ & $0.0 \%$ & $0.0 \%$ \\
\hline
\end{tabular}

Note: The mental model theoretical forecast with 1 explicit, 1 implicit mental model for $I F A$, then $B$ is according to Table 6 in Johnson-Laird et al. (1999, p. 73). The conjunction interpretation is equivalent to the representation of the conditional with only 1 explicit model. For the conjunction and disjunctions, the mental model theoretical predicted value and logical norm are identical. $\wedge$ denotes a conjunction and. $\neg$ is a tag representing negation.

Applying of the Mental Model Theory to score the responses and to infer the number of models: The predicted probability estimates listed in Table 2 consider possible interpretations 
of the conditional If $A$, then $B$. From Table 1 (akin to Johnson-Laird et al. (1999, Table 2)), we obtain the proposed models for the conditional: The fully explicit models epitomise the logic norm of formal rule theories describing If $A$, then $B$ as a material implication. The initial mental models represent the mental model theoretical possibilities, while conjunction values signify the conjunctive interpretation as one conjunction $A$ and $B$. Another possible interpretation of the conditional If $A$, then $B$ is a biconditional If, and only if $A$, then $B$; it produces the same forecast as the mental model theoretical initial models. Applying the Mental Model Theory of Extensional Reasoning to these premises, we can estimate how many models a respondent considers to make a likelihood judgement. We infer the type of mental representation from the observed answer by working backwards through the proportionality and equiprobability principles, postulating an interpretation of the premise consistent with the observed answer and score.

\section{Method}

\section{Participants}

The 58 first year students who participated in this experiment in exchange for course credits are from the faculty of psychology, University of Leuven. They were tested in groups of up to 30 people. Participants' mean age is 19 years $(S D=3.76$, skewness $=5.09)$.

\section{Task}

The four premises used in the first experiment are phrased as follows:

AND There is a box in which there is a yellow card and a green card.

XOR There is a box in which there is a yellow card, or a green card, but not both.

OR There is a box in which there is a yellow card, or a green card, or both.

IF There is a box in which if there is a yellow card then there is a green card.

Each premise is combined with four problems:

A1) In the box there is at least a yellow card.

A2) In the box there is a yellow card and a green card.

A3) In the box there is a yellow card and there is not a green card.

A5) In the box there is neither a yellow card nor a green card.

In case of the conditional premise, a fifth problem was posed to the participants:

A4) In the box there is a green card and there is not a yellow card.

The objects mentioned in the questions are either a ball, card, flower, marble, cup or tree. Only one type of object is mentioned within a given question. The colour attributes use two of the following colours: blue, green, black, brown, yellow, pink, red, white. Unusual object-colour combinations are not used. All objects are presented as being in a box, except for the tree that stands in a garden.

In the graphical input condition, participants mark each answer on a separate scale with following features: a horizontal line with eleven tick-marks above it, labelled from $0 \%$ to $100 \%$ in steps of $10 \%$. Adjacent to and below the horizontal line, a horizontal bar divides the scale into three sections of length $1 / 3$ with the values $1 / 3$ and ${ }^{2} / 3$ labelled. Next below that bar is a sec- 
ond horizontal bar dividing the scale into four sections of length $1 /{ }_{4}$, labelled at $1 / 4,1 /{ }_{2}$ and $3 / 4$.

\section{Measurements}

Using the norms listed in Table 2 as target values (logical norm or mental model theoretical forecast), we count the number of correct responses and compare our results with those published by Johnson-Laird et al. (1999). To count correct answers, we consider any response that fell on the norm or within a $\pm 4 \%$ band (including the limits of the range) to be at that norm. This is more narrow than the $\pm 5 \%$ band used in the 1999 original paper, yet it avoids any overlap between the $1 / 3$ and the $1 / 4$ values. For the end points of the scale, we apply a bin width of 5\%: e.g. in case the norm is zero, any response in the range $0 \%$ up to and including $5 \%$ is counted as correct.

We shall not look at merely the average of the given responses because of the spread and large variance observed on some premises. Answers get scored in following categories: logically correct, mental model theoretically predicted, where applicable conjunction interpretation or biconditional interpretation, incorrect or no-clue. We keep track of response entry mode and of the questions' formulation with objects and attributes. Parameters we monitor are the entry format of responses, participants' gender, the extent that participants use the no-clue outlet to signal that they can not find an answer, and whenever a contradiction arises between the premise and problem item.

\section{Procedure}

Written instructions given to the participants include a cover sheet with a graphical scale and two practice problems. Participants are asked to mark their responses on each entry field of the series of problems provided. In the graphical entry condition, participants must mark a point directly with the pencil on the scale on their paper questionnaire and not mark a range. Each premise is displayed on a separate page, with the order of presentation being randomized. The problems are listed after each premise on the same page with random rotation of the order of listing; below each problem, either the entry field or scale is printed. Participants have to work through the problems in the order presented. They can take as much time as they need and are reminded in the instructions to consider all information provided in a given problem. The experiment is conducted under the supervision of two experimenters. Participants receive no feedback about their answers. We run the experiment in Dutch, the native language of participants.

\section{Results}

Our analysis starts by showing that the answers are significantly different from random guesses. We then score the data against predicted responses, considering different possible interpretations of the premises and take a detailed look at the distribution of responses. We also assess the impact of entry mode and apply linear modelling to estimate the effect of premise type on accuracy.

Participants perform better than chance and their responses are significantly different from 
random guesses. The $\chi^{2}$ statistic of the random benchmark compared with the index composed of the sum of the scores of items is 90.9 on $4 \mathrm{df}$ with $p<2.2 * 10^{-16}$ for the xor exclusive disjunction; for the or inclusive disjunction: $\chi^{2}(4)=53.3$ with $p=7.29 * 10^{-11}$; for the If conditional $\chi^{2}(4)=59.7$ with $p=3.35 * 10^{-12}$. The random benchmark we use to compare the observed distribution of index scores per premise is the binomial distribution with $a^{9} / 101$ chance of guessing correctly per item comprised in the index ${ }^{1}$. The high $\chi^{2}$ values and the number of observations point towards large effect sizes according to Cohen (1988, p. 216) and to a high statistical power, indicating that the responses are very different from random guesses.

Conjunction, Exclusive and Inclusive Disjunction: The Exclusive Disjunction $A$ xor $B$ section in Table 3 compares our results with the Table 4 in Johnson-Laird et al. (1999, p. 72). The Inclusive Disjunction $A$ or $B$ section in Table 3 is to be compared with Table 5 in Johnson-Laird et al. (1999, p. 73). The Conditional If $A$, then $B$ section in Table 3 is to be compared with Table 6 in Johnson-Laird et al. (1999, p. 73). The mean responses we observe are similar to the reported means; however, a closer look at the distributions of respondents' probability estimates yields a more differentiated picture.

(Insert Table 3 about here)

The mean of responses presented in Table 3 coincides with the prediction of the Mental Model Theory: e.g. problem $p(A)$ with premise $A$ or $B$, the mean response is $61.6 \%$ against a mental model norm of $66.6 \%$, however only $23.2 \%$ of responses fall on or near the norm; the remaining answers are distributed around the values $25 \%, 50 \%, 75 \%, 100 \%$. For the problem $p(\neg A$ and $\neg B)$ combined with the premise If $A$, then $B$, the mean response is $51.6 \%$, close to the mental model norm. However, only $30.9 \%$ of responses to $p(\neg A$ and $\neg B)$ are on the mental model norm. The distributions of responses in the histograms in Figure 1 show a wide spread of estimated probabilities and complement earlier analyses.

(Insert Figure 1 about here)

Conditional: The conditional warrants a refined analysis based on differing interpretations to explain performance on the five problem-items. For example, in the conditional If $A$, then $B$ with problem $p(A$ and $B)$ : A third of responses fall on the value $100 \%$, which is indicative of a conjunction interpretation; another third of responses are at the $50 \%$ value, along the mental model norm that also corresponds to a biconditional interpretation; only one in eight respondents consider the three possibilities and answer around the one-third logical norm. Although the pattern of norms is identical for the items $p(A$ and $B)$ and $p(A)$, yet the fraction of zero answers at $p(A)$ is higher than at $p(A$ and $B)$ probably because $p(A)$, which is presented as $p$ (at least $A)$, could be interpreted as $p(A$ and not- $B)$. In Figure 1, the histogram of If $A$, then $B$ combined with $p(A$ and $\neg B$ ) shows $84.5 \%$ of correct responses. The statement $A$ and $\neg B$ contradicts the premise If $A$, then $B$, and all interpretations predict the answer $0 \%$. Besides the Mental Model Theory which accurately predicts this pattern, the detection and resolution of the contradiction yields

\footnotetext{
${ }^{1}$ The four items included in the index are $p(A), p(A$ and $B), p(A$ and $\neg B)$ and $p(\neg A$ and $\neg B)$.
} 
the same predicted answer. The $A$ and $\neg B$ statement is not one of the initial or explicit mental models of any interpretation.

Performance by Premise: In building a linear model (statistical analysis conducted in R (Ihaka \& Gentleman, 1996)), we focus on the number and complexity of mental representations as the theoretically most relevant factor to account for variation. The linear model features response-accuracy as the dependent variable along with three predictors: the type of premise, the mode of entry and item-specific differences (for control). Participants' gender is excluded because it has no significant effect. A logistic regression model is fitted to all data from this first experiment. The difference between the conjunction $A$ and $B$ and the exclusive disjunction $A$ xor $B$ premise is not significant $(z=-1.430, p=0.1528)$. Relative to the conjunction $A$ and $B$, the other premises $A$ or $B$ and If $A$, then $B$ exhibit a decrease in performance: For the inclusive disjunction $A$ or $B$, the model forecasts a $73.8 \%$ drop in the odds of answering the question logically $\left(z=-6.839, p=7.95 * 10^{-12}\right)$. For the conditional If $A$, then $B$, the drop in odds of answering logically is $91.3 \%\left(z=-11.774, p<2 * 10^{-16}\right)$. The differences are statistically significant when we compare the two easiest connectives to the more difficult $A$ or $B$ inclusive disjunction and the If $A$, then $B$ conditional. The type of premise is a highly ${ }^{2}$ significant factor in explaining performance along the logical norm. The pattern of increasing difficulty is confirmed by the modified and expanded linear models that we tested.

Method of Eliciting Probability Estimates The two modes (graphical versus numerical entry) of elicitation of probability have a small but significant impact on the accuracy of responses, and on the use of the no-clue field to avoid guessed responses. The effect on the logical accuracy of responses is a higher score of $62.8 \%(95 \% \mathrm{CI}[58.8,66.6])$ in the numerical entry mode versus $55.2 \%$ (95\% CI[50.8, 59.5]) in graphical entry. The logistic regression model estimates that switching from graphical mode to numerical entry mode improves the odds of answering a question logically by $36 \%(z=2.59, p=0.0096)$. The $\chi^{2}(1)$ effect size is small $(w=0.0767$, $\chi^{2}(1)=6.418, p=0.0113$ ). Effect size (and power) values are derived from Cohen (1988, p. 221). The range $[50.8 \%, 66.6 \%]$ covered by the logical accuracy estimates of the two input modes is wider than the confidence interval of the pooled data ( $\mathrm{M}=59.3 \%, 95 \% \mathrm{CI}[56.4,62.2])$. The number of no-clue answers was 17 in the numerical entry condition and zero in the graphical entry mode. In the aggregate pattern of responses, we see that respondents in the graphical entry mode tend to shy away from marking their answer on the edge of the scale (at $0 \%$ and $100 \%$ ). We make this comparison after adjusting for the number of participants in each condition and verifying that the distribution of norms is equivalent in both graphical and numerical entry conditions.

\footnotetext{
${ }^{2}$ In the analysis of variance, the type of premise factor has an F-value $=76.32$ with $p<2.2 * 10^{-16}$. We use factorial ANOVA for unordered predictors.
} 


\section{Discussion}

We survey the following research question based on the results of Experiment 1: the scope of application of the Mental Model Theory and the explanatory power of the four interpretations of the conditional; the parsimonious build-up of models and the relative difficulty of premises; the questions of reasoning strategy, conjunction fallacy, and of the method of eliciting probability estimates.

Scope of Application of the Theory: To compare the explanatory power of different theories, we evaluate participants' responses to the conditional premise If $A$, then $B$ according to three possible interpretations described by Evans et al. (2007): the logical norm or indicative material implication, the mental model theoretical norm (which in this experimental tasks is equivalent to a biconditional interpretation), and the conjunction interpretation. Applying this comparative scoring to the four premises shows that the Mental Model Theory can account for up to 65\% of the data, while the logical norm accounts for $59.3 \%$ of all the answers collected in this first experiment. The difference stems from the interpretation of the conditional: The Mental Model Theory can explain $42.4 \%$ of the illogical answers, more than the conjunctive interpretation which accounts for less than a third of the illogical answers.

The $42.4 \%$ illogical answers explained by the Mental Model Theory illustrate the parsimonious build-up of an incomplete representation that considers too few possibilities, which is a distinctive trait of bounded rationality. Summing-up the individual scores on the five problems listed in Table 2 for the conditional If $A$, then $B$ reveals that the values predicted by the Mental Model Theory explain $47.8 \%$ of the data (the same holds for the biconditional interpretation); conjunctive interpretations account for $39.6 \%$, and logic explains $28.8 \%$. We conclude that for the estimation of probabilities using premises mentioning two objects, the Mental Model Theory has a higher predictive power than the conjunctive or logical accounts.

The relative difficulty of premises is approximated by the rate of incorrect answers: The rate of incorrect responses increases as we shift from the easier propositional connectives, the conjunction $A$ and $B$, to the progressively more difficult premises, the exclusive disjunction $A$ xor $B$, the $A$ or $B$ disjunction and the If $A$, then $B$ conditional. Our data confirm earlier studies of deductive reasoning with these connectives (Johnson-Laird \& Byrne, 1991).

Reasoning Strategy: The extensional reasoning strategy explains most of the responses observed. Yet an alternative strategy, the detection of contradictions based on conflict monitoring, also explains the answers observed in cases where the problem item stands in contradiction to the premise. Performance is consistently higher where the problem contradicts the premise. This observation applies to the disjunctions and the conditional. The highest scores occur when the mental model theoretical forecast is at zero, with zero probability indicating a (perceived) contradiction. This can be explained by a respondent's detection of a conflict in the information presented: As some of these contradictions are obvious to the respondent, the answer is straightforward. In these contradicting cases, people who build their conclusion on mental models arrive at the same answer with a conflict detection strategy or an extensional reasoning 
strategy. A conflict monitoring process used to detect the contradiction could trigger a change in cognitive control, altering the strategy in favour of a simple approach: If the problem is impossible based on the premise, its chance of occurrence is zero.

Conjunction Fallacy: Correct inclusion judgments of the probability of a constituent $p(A)$ $\geq p(A$ and $B)$ range from $83.9 \%$ to $89.3 \%$. This shows that respondents eschew the conjunction fallacy and is in line with the observation made by Johnson-Laird et al. (1999, p. 71) that $92.3 \%$ avoid this fallacy. The observed incidence of conjunction fallacies is much lower than the $50 \%$ rate we expect from the random guessing of probability estimates.

Method of Eliciting Probability Estimates: The 1.5\% incidence of no-clue answers in this first experiment is low. Most no-clue answers (12 out of 17) are given in response to the conditional If $A$, then $B$, indicating that participants experience more difficulty with the conditional than with the three other premises. With all no-clue answers recorded in the numerical entry mode, participants working in the graphical entry mode answered all problems, albeit with a lower score. The lower rate of use of the no-clue field in the graphical entry mode does not suffice to explain the difference in logical accuracy between the numerical and graphical entry modes. Assuming that the incidence of no-clue responses observed in the numerical entry mode were applied to the graphical entry mode, a reduction of the difference in logical accuracy by 1.8 percentage points (i.e. $28 \%$ of the difference) could be ascribed to the use of the no-clue field. We observe a small effect of the entry mode on the distribution of responses: Participants in the graphical entry condition tend to shy away from the edges of the scale, that is the zero and $100 \%$ values. As unveiled by the use of two entry modes, the variance in our estimates is large: Confidence intervals for the estimate of logical accuracy range from one-half to twothirds. Respondents are influenced by other factors including the way of giving their answer. We maintain the two entry modes in Experiment 2 with more difficult problems to see if this effect is replicated or magnified. 
Table 3

Responses (\%) on the Norm (\%) for $A$ and $B, A$ xor $B, A$ or $B$ and If $A$, then $B$.

\begin{tabular}{|c|c|c|c|c|c|c|}
\hline \multirow{2}{*}{\multicolumn{2}{|c|}{$\begin{array}{l}\text { Premise } \\
\text { Source Measure }\end{array}$}} & \multicolumn{5}{|c|}{ Item } \\
\hline & & \multirow[t]{2}{*}{$\mathrm{p}(\mathrm{A})$} & \multirow[t]{2}{*}{$\mathrm{p}(\mathrm{A}$ and $\mathrm{B})$} & \multirow[t]{2}{*}{$\mathrm{p}(\mathrm{A}$ and $\neg \mathrm{B})$} & \multirow[t]{2}{*}{$\mathrm{p}(\neg \mathrm{A}$ and $\mathrm{B})$} & \multirow[t]{2}{*}{$\mathrm{p}(\neg \mathrm{A}$ and $\neg \mathrm{B})$} \\
\hline \multicolumn{2}{|c|}{ Conjunction $A$ and $B$} & & & & & \\
\hline & MM-norm & 100.0 & 100.0 & 0.0 & 0.0 & 0.0 \\
\hline 1999 & reported mean & NA & NA & NA & NA & NA \\
\hline Exp.1 & mean response & 87.1 & 93.7 & 7.1 & 8.0 & 8.4 \\
\hline Exp.1 & variance & 6.2 & 3.9 & 3.8 & 4.2 & 4.4 \\
\hline Exp.1 & MMforecast & $70.2(\mathrm{n}=57)$ & $84.5(n=58)$ & $81.0(\mathrm{n}=58)$ & $79.3(\mathrm{n}=58)$ & $82.8(n=58)$ \\
\hline 1999 & MMforecast & NA & NA & NA & NA & NA \\
\hline Exp.1 & no-clue's & $\mathrm{nc}=1$ & $\mathrm{nc}=0$ & $\mathrm{nc}=0$ & $\mathrm{nc}=0$ & $\mathrm{nc}=0$ \\
\hline \multicolumn{7}{|c|}{ Exclusive Disjunction $A$ xor $B$} \\
\hline & MM-norm & 50.0 & 0.0 & 50.0 & 50.0 & 0.0 \\
\hline 1999 & reported mean & 45.0 & 6.0 & 53.0 & NA & 16.0 \\
\hline Exp.1 & mean response & 46.2 & 0.3 & 62.0 & 62.0 & 10.4 \\
\hline Exp.1 & variance & 7.4 & 0.0 & 4.7 & 4.7 & 5.1 \\
\hline Exp.1 & MM Forecast & $57.9(\mathrm{n}=57)$ & $98.3(\mathrm{n}=58)$ & $69.0(n=58)$ & $69.0(\mathrm{n}=58)$ & $79.3(\mathrm{n}=58)$ \\
\hline 1999 & MM Forecast & $72.7(n=22)$ & $86.4(n=22)$ & $81.8(\mathrm{n}=22)$ & NA & $72.7(n=22)$ \\
\hline Exp.1 & no-clue's & $\mathrm{nc}=1$ & $\mathrm{nc}=0$ & $\mathrm{nc}=0$ & $\mathrm{nc}=0$ & $\mathrm{nc}=0$ \\
\hline \multicolumn{7}{|c|}{ Inclusive Disjunction $A O R B$} \\
\hline & MM-norm & 66.6 & 33.3 & 33.3 & 33.3 & 0 \\
\hline 1999 & reported mean & 60.0 & 45.0 & 44.0 & NA & 7.0 \\
\hline Exp. 1 & mean response & 61.6 & 53.0 & 49.9 & 50.7 & 8.1 \\
\hline Exp. 1 & variance & 7.4 & 8.5 & 8.4 & 8.4 & 5.5 \\
\hline Exp. 1 & MM Forecast & $23.2(n=56)$ & $49.1(n=57)$ & $53.4(n=58)$ & $51.7(\mathrm{n}=58)$ & $86.2(n=58)$ \\
\hline 1999 & MM Forecast & $40.9(n=22)$ & $68.2(n=22)$ & $63.6(n=22)$ & NA & $90.9(n=22)$ \\
\hline Exp.1 & no-clue's & $\mathrm{nc}=2$ & $\mathrm{nc}=1$ & $\mathrm{nc}=0$ & $\mathrm{nc}=0$ & $\mathrm{nc}=0$ \\
\hline \multicolumn{7}{|c|}{ Conditional If $A$, then $B$} \\
\hline & MM-norm & 50.0 & 50.0 & 0.0 & 0.0 & 50.0 \\
\hline 1999 & reported mean & 58.0 & 68.0 & 0.0 & NA & 38.0 \\
\hline Exp.1 & mean response & 50.7 & 67.7 & 6.3 & 29.3 & 51.6 \\
\hline Exp.1 & variance & 8.5 & 7.2 & 2.4 & 10.2 & 11.1 \\
\hline Exp.1 & MM Forecast & $43.6(n=55)$ & $32.1(\mathrm{n}=56)$ & $84.5(n=58)$ & $46.3(n=54)$ & $30.9(n=55)$ \\
\hline 1999 & MM Forecast & $63.6(n=22)$ & $54.5(n=22)$ & $100.0(n=22)$ & NA & $54.5(n=22)$ \\
\hline Exp.1 & Logic norm & 12.7 & 12.5 & 84.5 & 13.0 & 18.2 \\
\hline Exp.1 & Conjunction I & 16.4 & 33.9 & 84.5 & 46.3 & 14.5 \\
\hline Exp.1 & no-clue's & $\mathrm{nc}=3$ & $\mathrm{nc}=2$ & $\mathrm{nc}=0$ & $\mathrm{nc}=4$ & $\mathrm{nc}=3$ \\
\hline
\end{tabular}

Note. 'MM Forecast' stands for the mental model theoretical predicted answer, which in the case of the If $A$, then $B$ is equivalent to a bi-conditional interpretation. The conjunction interpretation of the conditional is equivalent to building just one explicit mental model. For the conditional with $p(A$ and $\neg B$ ), all norms consider 0 as correct. $\neg$ is a tag representing negation. $\quad(\mathrm{n}=\quad)$ indicates the total number of respondents for each item on this premise. 
Figure 1

Histogram for Conditional Premise If $A$, then $B$
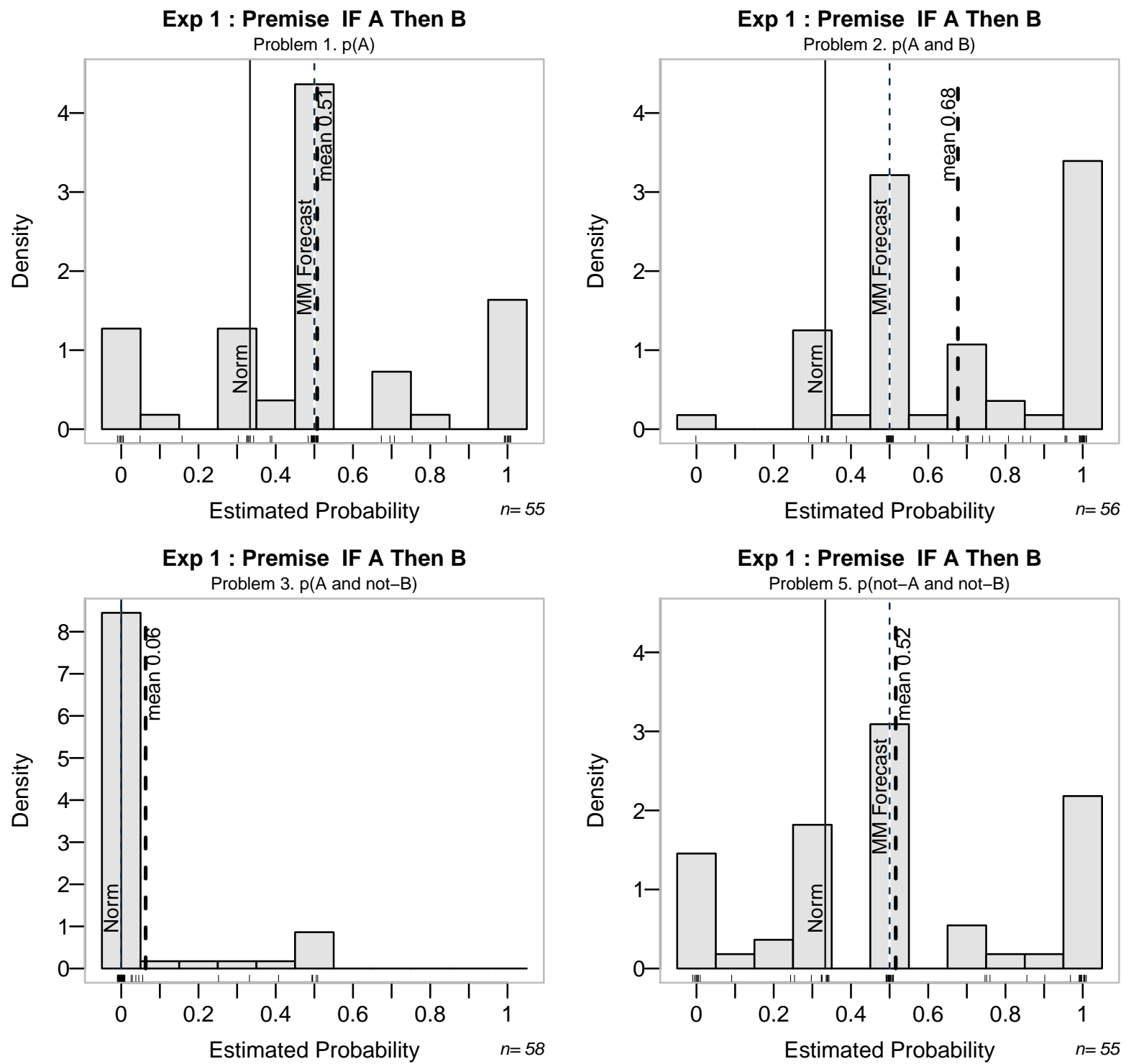

Overlayed norms: Norm= logical norm; $M M$ Forecast $=$ mental model theoretical interpretation when different from logical norm. Mean= mean of estimated probabilities. 


\section{Experiment 2: A Bias in Extensional Reasoning}

The Mental Model Theory forecast emerges as the better predictor of responses to the conditional premise in Experiment 1 (Table 3): The results are consistent with an explanation that participants relied on simple mental representations, resulting in a bias in favour of the mental model theoretical and conjunctive interpretation of the conditional. Experiment 2 focuses on the bias away from logical rules in the direction of the mental model theoretical forecast and measures its extent. We examine situations where the answers predicted by the Mental Model Theory differ from the logical norm. The difference between the forecasts stems from the extension of models: While logical rule theories require the build-up of complete representations, fleshing out all models, the Mental Model Theory predicts that only a subset of all possible models is actually built.

Our approach follows Johnson-Laird et al. (1999) who use combinations of nested disjunctions, the conjunction and the conditional to formulate two sets of problems: The test group answers problems that produce predictions which clearly separate the logical from the mental model theoretical interpretation, while the control group answers a set of matched problems where all forecasts of the different interpretations are identical. This allows us to compare the predictive power of the Mental Model Theory forecasts of several interpretations against logical rules. Because two of the premises include conditionals, the mental model theoretical, conjunctive, logical and biconditional interpretations of conditionals and their resulting description with models is relevant to our analysis. These different interpretations produce varying estimates of probabilities as shown in Table 4. Given our findings in Experiment 1, we expect a similar pattern in Experiment 2. Hence, we shall concentrate on the effect of different interpretations.

We present the differences in interpretation and the resulting mental representation for a premise with a conditional and disjunction: If $A$, then either $B$ or $C$, not all three. Taking this premise as an example, we throw light on how a reasoner builds-up mental representations of a conditional with a nested disjunction. As shown in Table 7, for premise E3 If A, then either $B$ or $C$, not all three, the two initial models correspond to just a third of the fully explicit representation.

The process of mental model build-up comprises steps to flesh-out or drop certain models and represent explicitly as little information as possible: "Models can contain abstract symbols that do not directly correspond to anything in the physical world" (Johnson-Laird et al., 1992, p. 421). When the implicit representation ... is dropped from the initial mental models of the If $A$, then $B$ conditional, the result is a conjunction interpretation of that same conditional (see Table 1). The Mental Model Theory postulates that this conjunction interpretation is applied to premise E3: "Because the premises containing conditionals also include a disjunction, the implicit model of a conditional should tend to be forgotten" (Johnson-Laird et al., 1999, p. 75). Therefore, predictions according to the mental model theoretical and conjunction interpretations that do not take implicit models into account are equivalent.

To explain the two-model build-up characteristic of the mental model theoretical interpretation, we assume that respondents start with the conditional If $A$, then $Q$, holding $\mathrm{Q}$ as a symbol 
for either $B$ or $C$, and in a second step incorporate the disjunction $B$ xor $C$ by fleshing out the first initial model and drop the implicit ellipsis ... (see Table 7). The biconditional interpretation yields four models: The conditional If $A$, then $Q$ is first represented by two explicit models, which, in turn, are fleshed-out with two models each to incorporate the disjunction. An alternative build-up is the conditional with true consequent only: Both the first initial model and the implicit model are expanded with two models of the disjunction that make the consequent of the conditional true, leading to four models.

Finally, the logic-interpretation entails a build-up of models of all six logically true combinations of the conditional and inclusive disjunction (see the fully explicit models in Table 7 and Table 8). These different representations allow us to predict responses for each type of interpretation.

In this experiment, we make two changes compared to the approach taken by Johnson-Laird et al. (1999): We apply a between-subjects design for the test and control condition (instead of letting participants act as their own controls), and translate the questions into Dutch, the native language of the participants in Experiment 2.

\section{Method}

\section{Participants}

First year students at the faculty of psychology, University of Leuven, participated in this experiment in exchange for course credits. They were tested in groups of up to 30 people. In the test group, the 54 participants' mean age is 19 years $(\mathrm{SD}=3.52$, skewness $=5.93)$. The number of participants in the control group is 48 , with mean age 19.4 years $(\mathrm{SD}=4.57$, skewness $=3.87)$.

\section{Task}

The premises used by the test group have the potential of a bias towards one of several interpretations: mental model theoretical, biconditional and logical. These premises are

E1. A or both B and C, not all three

E2. A or else if B then $\mathrm{C}$, not all three.

E3. If $\mathrm{A}$, then either $\mathrm{B}$ or $\mathrm{C}$, not all three.

The control group premises, which are unbiased, are

C1. A and either B or else C, not all three

C2. A or else $\mathrm{B}$ or else $\mathrm{C}$, not more than one

C3. A or else B, and C, not all three.

Each premise is matched with three probability estimating tasks out of following set: 1.p(A and B), 2.p(A and C), 3.p(B and C), 4.p(only A), 5.p(empty box), S6) p(at least A), 7.p(at least B). The assignment of items per premise is summarised in Table 4. 
Table 4

Assignment of Items and Norms per Premise in Experiment 2

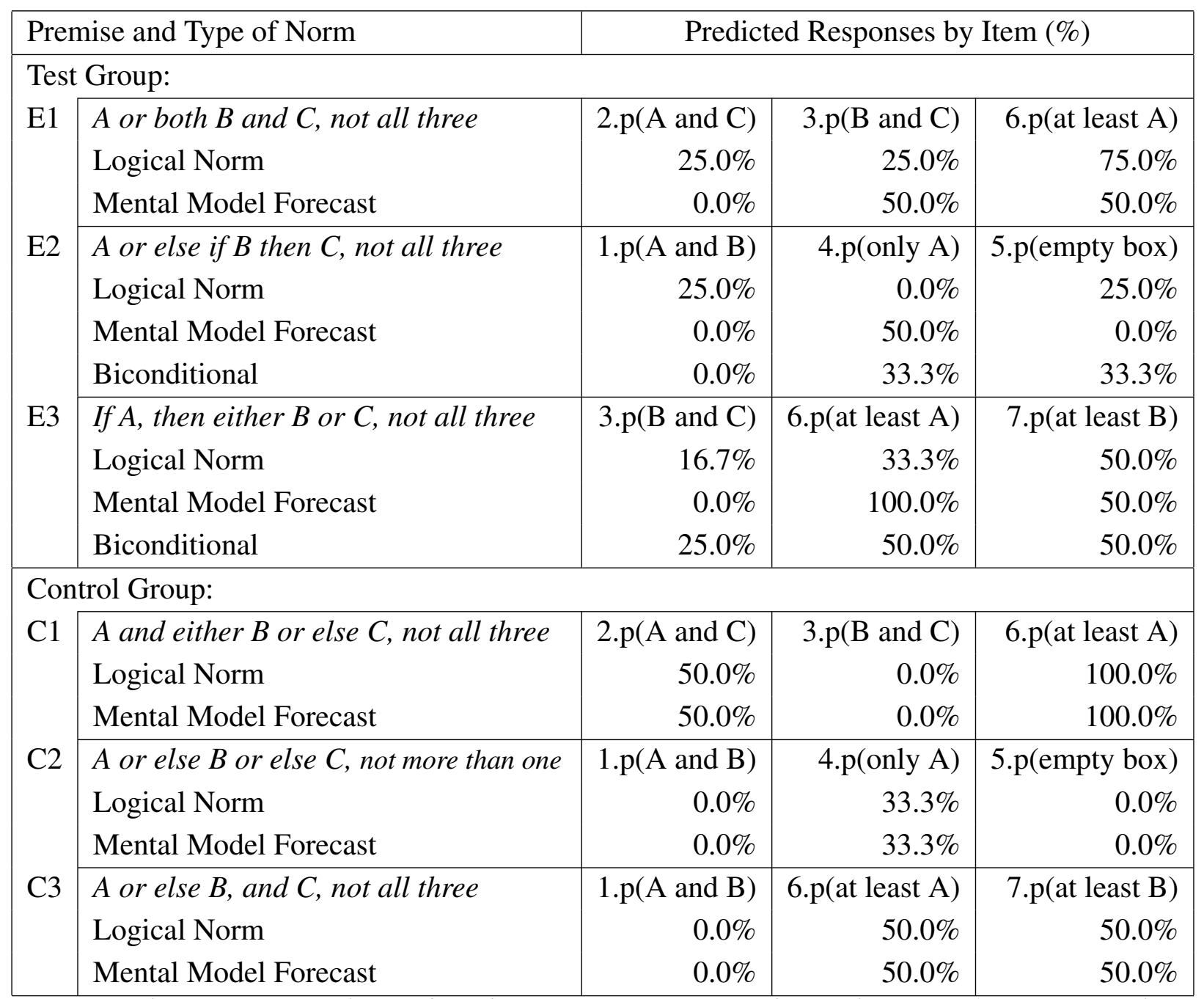

Note: In the test group, the conjunction interpretation produces the same forecasts as the mental model theoretical interpretation. In the control group, all interpretations yield the same forecast.

(Insert Table 4 about here)

The predicted values are derived from the mental models listed in Table 8 in Appendix A. Depending on the mental model theoretical or biconditional interpretation of test group premises, the number of initial mental models differs from the fully explicit models of the logical interpretation. The objects mentioned in the questions are either a ball, card, flower, marble, cup or tree. Only one type of object is mentioned within a given question. The colour attributes use three of the following colours: blue, green, black, brown, yellow, pink, red, white. Unusual object-colour combinations are avoided. Objects are presented as being in a box, except for the tree which stands in a garden. To assert the clarity ${ }^{3}$ of such abstract premises, we conducted a survey with incoming students at the University of Leuven (Chevalley \& Schaeken, 2013), and

\footnotetext{
${ }^{3}$ As a reviewer of the article by Johnson-Laird et al. (1999, p. 74) wrote "It is difficult for me to believe that a random sample of Chicagoans, for example, would have responded to such an ill-defined question with much more than a blank stare".
} 
concluded that the majority of participants are able to understand these premises correctly.

\section{Measurements}

Accuracy of responses is assessed in the same way as in Experiment 1, by tallying the totals that are at or near the forecasted answer of one of the interpretations. We track the same parameters as in Experiment 1.

\section{Procedure}

The procedure of presenting the instructions, the premises and problems is the same as in Experiment 1. Each participant gets either the three test premises or the three control premises. Each premise is presented on one page together with three problem items. Table 4 shows the predicted responses for each interpretation. Participants are asked to write their response on each entry field provided, and in the graphical entry mode, to mark a point on the graphic scale. We introduce the same modifications in the experimental procedure, compared to the original experiment (Johnson-Laird et al., 1999, p. 75), as in Experiment 1: A no-clue field is added to reduce the occurrence of pure guesses and we run the experiments in Dutch, the native language of participants.

\section{Results}

Using the norms listed in Table 4, we tally the number of responses falling on each norm and compare our results to Johnson-Laird et al. (1999, p. 88). Results for the items of test premises are listed in Table 5: The conjunctive and mental model theoretical interpretations overlap. (Insert Table 5 about here)

Observed responses are significantly different from random guesses, as was the case in the original experiment: The $\chi^{2}$ statistic of the index composed of scores on the Mental Model Theory forecast for three items per premise compared to a random benchmark confirms the difference. For premise E1 A xor $(B$ and $C), \chi^{2}(3)=42.92$, with $p=2.56 * 10^{-9}$. For E2 $A$ or else if $B$ then $C, \chi^{2}(3)=84.31, p<2.2 * 10^{-16}$. For E3 If $A$, then $(B$ xor $C), \chi^{2}(3)=27.67$, $p=4.27 * 10^{-6}$. These statistics imply large effect sizes and power-values close to one. When applied to the unbiased answers (logical norm) in the test condition, the $\chi^{2}$ statistic does not reveal any significant difference from the random benchmark. This is due to the very low scores observed on the logical norm in the test condition (see Table 5). Low scores for logical inferences in the test condition are also reported by Johnson-Laird et al. (1999, p. 75).

\section{(Insert Table 6 about here)}

In the control condition, observed responses are significantly different from random guesses: For $\mathrm{C} 1 \mathrm{~A}$ and $(B$ xor $C): \chi^{2}(d f=3)=47.111, p=3.292 * 10^{-10}$. For $\mathrm{C} 2 \mathrm{~A}$ xor $B$ xor $C: \chi^{2}(3)=70.4176, p=3.474 * 10^{-15}$. For $\mathrm{C} 3\left(A\right.$ xor B) and $C: \chi^{2}(3)=40.5259$ with $p=8.243 * 10^{-9}$. These values point towards large effect sizes (Cohen, 1988, p. 216+223) confirming that responses are different from guesses.

(Insert Figure 2 about here) 
Table 5

Responses by Norm for Test Premises E1. A xor (B and $C)$, E2. A or else if $B$ then $C, \mathrm{E} 3$. If $A$ then either ( $B$ or $C$ ).

\begin{tabular}{|c|c|c|c|c|}
\hline Source & Premise and Measure & & Item & \\
\hline E1. & $A$ xor $(B$ and $C)$ & 2.p(A and $C)$ & 3.p(B and $C)$ & 6.p(at least A) \\
\hline Exp.2 & Fraction at MM Forecast & $44.2 \%(\mathrm{n}=52)$ & $59.3 \%(\mathrm{n}=54)$ & $45.3 \%(n=53)$ \\
\hline JL1999 & Fraction at MM Forecast & $72.0 \%(\mathrm{n}=25)$ & $68.0 \%(\mathrm{n}=25)$ & $68.0 \%(n=25)$ \\
\hline Exp.2 & At Logic Norm, unbiased & $15.4 \%(\mathrm{n}=52)$ & $7.4 \%(\mathrm{n}=54)$ & $7.5 \%(\mathrm{n}=53)$ \\
\hline JL1999 & At Logic Norm, unbiased & $8.0 \%(\mathrm{n}=25)$ & $0.0 \%(\mathrm{n}=25)$ & $0.0 \%(\mathrm{n}=25)$ \\
\hline Exp.2 & No-Clue answers & 2 & 0 & 1 \\
\hline Source & Premise and Measure & & Item & \\
\hline E2. & $A$ or else if $B$ then $C$ & 1.p $(A$ and $B)$ & 4.p(only A) & 5.p(empty box $)$ \\
\hline Exp.2 & Fraction at MM Forecast & $85.2 \%(\mathrm{n}=54)$ & $75.9 \%(\mathrm{n}=54)$ & $83.0 \%(\mathrm{n}=53)$ \\
\hline JL1999 & Fraction at MM Forecast & $76.0 \%(\mathrm{n}=25)$ & $56.0 \%(\mathrm{n}=25)$ & $68.0 \%(n=25)$ \\
\hline Exp.2 & At Logic Norm & $5.6 \%(n=54)$ & $1.9 \%(n=54)$ & $3.8 \%(\mathrm{n}=53)$ \\
\hline JL1999 & At Logic Norm & $0.0 \%(\mathrm{n}=25)$ & $20.0 \%(\mathrm{n}=25)$ & $4.0 \%(\mathrm{n}=25)$ \\
\hline Exp.2 & Biconditional & $85.2 \%(n=54)$ & $3.7 \%(\mathrm{n}=54)$ & $7.5 \%(\mathrm{n}=53)$ \\
\hline Exp.2 & Conjunction & $85.2 \%(\mathrm{n}=54)$ & $75.9 \%(\mathrm{n}=54)$ & $83.0 \%(n=53)$ \\
\hline Exp.2 & No-Clue answers & 0 & 0 & 1 \\
\hline Source & Premise and Measure & & Item & \\
\hline E3. & If $A$ then either ( $B$ or $C$ ) & 3.p(B and $\mathrm{C})$ & 6.p(at least A) & 7.p(at least B) \\
\hline Exp.2 & Fraction at MM Forecast & $59.6 \%(\mathrm{n}=52)$ & $15.1 \%(\mathrm{n}=53)$ & $24.1 \%(n=54)$ \\
\hline 1999 & Fraction at MM Forecast & $56.0 \%(\mathrm{n}=25)$ & $48.0 \%(\mathrm{n}=25)$ & $64.0 \%(n=25)$ \\
\hline Exp.2 & At Logic Norm & $7.7 \%(\mathrm{n}=52)$ & $7.5 \%(\mathrm{n}=53)$ & $*$ \\
\hline 1999 & At Logic Norm & $0.0 \%(\mathrm{n}=25)$ & $4.0 \%(n=25)$ & $*$ \\
\hline Exp.2 & Biconditional & $1.9 \%(\mathrm{n}=52)$ & $34.0 \%(n=53)$ & * \\
\hline Exp.2 & Conjunction & $59.6 \%(\mathrm{n}=52)$ & $15.1 \%(\mathrm{n}=53)$ & * \\
\hline Exp.2 & No-Clue answers & 2 & 1 & 0 \\
\hline
\end{tabular}

Figure 2 highlights the difference between the bias in the test condition (left column) and the control condition (right column). In the top row, E1 $A$ xor ( $B$ and $C$ ) compares with $\mathrm{C} 1$ $A$ and (B xor $C)$, both with the probability estimation problem $p(B$ and $C)$. The test condition in the upper left chart shows the divergence between the logical norm at $25 \%$ and the mental model forecast at 50\%: The majority of responses clusters around the mental model forecasted value. On the right hand side, the control problem shows one norm at zero, with the majority of responses falling on this value. This comparison illustrates the difference in magnitude between responses based on a simplified representation and logical responses based on a fully explicit mental representation.

To assert the size of the bias towards simplified representations, we sum the number of logical responses in Table 5 (test) to compare it against the corresponding aggregate in Table 6 (con- 
Table 6

Responses for Control Premises $A$ and (B xor $C$ ), $A$ xor B xor $C, A$ xor ( $B$ and $C$ ).

\begin{tabular}{|c|c|c|c|c|}
\hline Source & Premise and Measure & & Item & \\
\hline $\mathrm{C} 1$. & $A$ and $(B$ xor $C)$ & 2.p $(A$ and $C)$ & 3.p(B and C) & 6.p(at least A) \\
\hline Exp.2 & Fraction at MM Forecast & $66.0 \%(n=47)$ & $77.1 \%(n=48)$ & $19.1 \%(n=47)$ \\
\hline 1999 & Fraction at MM Forecast & $56.0 \%(\mathrm{n}=25)$ & $44.0 \%(n=25)$ & $24.0 \%(n=25)$ \\
\hline Exp.2 & No-Clue answers & 1 & 0 & 1 \\
\hline Source & Premise and Measure & & Item & \\
\hline $\mathrm{C} 2$. & $A$ xor $B$ xor $C$ & 1.p $(A$ and $B)$ & 4.p(only A) & 5.p(empty box) \\
\hline Exp.2 & Fraction at MM Forecast & $91.7 \%(n=48)$ & $75.0 \%(n=48)$ & $66.0 \%(n=47)$ \\
\hline 1999 & Fraction at MM Forecast & $96.0 \%(n=25)$ & $44.0 \%(n=25)$ & $80.0 \%(n=25)$ \\
\hline Exp.2 & No-Clue answers & 0 & 0 & 1 \\
\hline Source & Premise and Measure & & Item & \\
\hline C3. & $A \operatorname{xor}(B$ and $C)$ & 1.p $(A$ and $B)$ & 6.p(at least A) & 7.p(at least B) \\
\hline Exp.2 & Fraction at MM Forecast & $85.1 \%(n=47)$ & $31.8 \%(n=44)$ & $32.6 \%(n=43)$ \\
\hline 1999 & Fraction at MM Forecast & $80.0 \%(n=25)$ & $68.0 \%(n=25)$ & $68.0 \%(n=25)$ \\
\hline Exp.2 & No-Clue answers & 1 & 4 & 5 \\
\hline
\end{tabular}

Note. Identical norms in the control condition: the logical answer and Mental Model Theory forecast are the same.

trol). The fraction of logical answers in the control condition is $61.1 \%$, and only $8.98 \%$ in the test condition. This drop is significant $\left(\chi^{2}(1)=271.0, p<2.2 * 10^{-16}\right)$ with a large effect size $w=0.55$. In contrast, the fraction of answers forecasted by the Mental Model Theory in the test condition is $54.7 \%$, a less significant drop $\left(\chi^{2}(1)=3.49, p=0.062\right)$ with a small effect size $w=0.065$. The contrast between the two effect sizes highlights that when respondents would need to distinguish a purely logical response from a response forecasted by the Mental Model Theory, as is the case in the test condition, the majority of their responses fall on the mental model theoretical forecast.

In the second row of Figure 2, we consider in detail premise E3 If $A$, then either ( $B$ or $C$ ) with the problem $p(A)$ : About one third of respondents estimate the probability to be $50 \%$, which is consistent with a biconditional interpretation of the conditional If $A$, then $Q$ (please refer to Table 7 for the different models and forecasted answers by type of interpretation). A biconditional interpretation implies a more complex mental representation than the conjunction interpretation of the conditional. 15\% of responses fall on the Mental Model Theory forecast of one, an answer that is also explained by the conjunction interpretation. Fewer than one in six responses is consistent with a conjunctive interpretation, a significant drop from the one out of three responses observed in Exp. 1 for If $A$, then $B$. Only 7.5\% give an unbiased response according to the logical norm. Confusion about the premise could also explain part of the responses at the $0 \%$ value, indicating that people think the information is in contradiction and the situation presented is impossible. In the aggregate over the three items presented with premise E3 If $A$ then either $B$ or $C$, not all three in Table 7, the 'Mental Model of conditional with implicit 
model retained' scenario explains 39\% of the total 159 responses, the Mental Model Theory interpretation (equivalent to the conjunction interpretation) $32.7 \%$, biconditional interpretation $20.1 \%$ and logic rules $13.2 \%$ of responses.

Table 7

Mental Models and Norms for Different Interpretations of the Premise E3 If A then either (B or C)

\begin{tabular}{|c|c|c|c|c|c|c|c|c|}
\hline \multirow{3}{*}{$\begin{array}{l}\text { Interpretation } \\
\text { Mental Model } \\
\text { Theoretical }\end{array}$} & \multicolumn{4}{|c|}{ Initial and Explicit Models } & \multicolumn{3}{|c|}{ Predicted Response (\%) } & \multirow{2}{*}{$\begin{array}{r}\text { Explains } \\
32.7 \%\end{array}$} \\
\hline & Q & A & $\mathrm{B}$ & $\neg \mathrm{C}$ & $\mathrm{p}(\mathrm{B}$ and $\mathrm{C})$ & $\mathrm{p}(\mathrm{A})$ & $\mathrm{p}(\mathrm{B})$ & \\
\hline & & A & $\neg \mathrm{B}$ & $\mathrm{C}$ & \multirow[t]{2}{*}{$0.0 \%$} & \multirow[t]{2}{*}{$100.0 \%$} & \multirow{2}{*}{$50.0 \%$} & \\
\hline \multirow[t]{2}{*}{ Conjunction } & A & A & $\mathrm{B}$ & $\neg \mathrm{C}$ & & & & \\
\hline & & A & $\neg \mathrm{B}$ & $\mathrm{C}$ & [31 ] & [8] & {$[13]$} & [ 52 ] \\
\hline \multirow[t]{4}{*}{ Biconditional } & Q & A & B & $\neg \mathrm{C}$ & $\mathrm{p}(\mathrm{B}$ and $\mathrm{C})$ & $\mathrm{p}(\mathrm{A})$ & $\mathrm{p}(\mathrm{B})$ & $20.1 \%$ \\
\hline & & A & $\neg \mathrm{B}$ & $\mathrm{C}$ & $25.0 \%$ & $50.0 \%$ & $50.0 \%$ & \\
\hline & $\neg \mathrm{Q}$ & $\neg \mathrm{A}$ & B & $\mathrm{C}$ & & & & \\
\hline & & $\neg \mathrm{A}$ & $\neg \mathrm{B}$ & $\neg \mathrm{C}$ & [1] & {$[18]$} & {$[13]$} & [32 ] \\
\hline \multirow{4}{*}{$\begin{array}{l}\text { Conditional } \\
\text { With True } \\
\text { Consequent } \\
\text { Only }\end{array}$} & Q & A & $\mathrm{B}$ & $\neg \mathrm{C}$ & $\mathrm{p}(\mathrm{B}$ and $\mathrm{C})$ & $\mathrm{p}(\mathrm{A})$ & $\mathrm{p}(\mathrm{B})$ & $39.0 \%$ \\
\hline & & A & $\neg \mathrm{B}$ & $\mathrm{C}$ & $0.0 \%$ & $50.0 \%$ & $50.0 \%$ & \\
\hline & . & $\neg \mathrm{A}$ & B & $\neg \mathrm{C}$ & & & & \\
\hline & & $\neg \mathrm{A}$ & $\neg \mathrm{B}$ & $\mathrm{C}$ & [31 ] & {$[18]$} & {$[13]$} & [62 ] \\
\hline \multirow{6}{*}{$\begin{array}{l}\text { Logical Rules } \\
\text { for the } \\
\text { Conditional }\end{array}$} & Q & A & B & $\neg \mathrm{C}$ & $\mathrm{p}(\mathrm{B}$ and $\mathrm{C})$ & $\mathrm{p}(\mathrm{A})$ & $\mathrm{p}(\mathrm{B})$ & $13.2 \%$ \\
\hline & & A & $\neg \mathrm{B}$ & $\mathrm{C}$ & $16.7 \%$ & $33.3 \%$ & $50.0 \%$ & \\
\hline & $\neg \mathrm{A}$ & $\neg \mathrm{A}$ & $\mathrm{B}$ & $\neg \mathrm{C}$ & & & & \\
\hline & & $\neg \mathrm{A}$ & $\neg \mathrm{B}$ & $\mathrm{C}$ & [4] & [4] & {$[13]$} & [ 21$]$ \\
\hline & $\neg \mathrm{Q}$ & $\neg \mathrm{A}$ & B & $\mathrm{C}$ & & & & \\
\hline & & $\neg \mathrm{A}$ & $\neg \mathrm{B}$ & $\neg \mathrm{C}$ & & & & \\
\hline \multicolumn{5}{|c|}{ Total Number of Responses } & 52 & 53 & 54 & 159 \\
\hline
\end{tabular}

Note. In this premise, the mental model theoretical forecast is equivalent to a conjunction interpretation of the conditional. Q is a placeholder for either $B$ or $C$. $\mathrm{p}(\mathrm{A})=\mathrm{p}$ (at least $\mathrm{A}) \cdot \mathrm{p}(\mathrm{B})=\mathrm{p}($ at least $\mathrm{B}) . \quad \neg$ is a tag representing negation. Explains refers to the fraction of the $\mathrm{N}=159$ responses that is explained by a particular interpretation. The number of responses that fall on a particular forecast are given in [brackets].

\section{(Insert Table 7 about here)}

The explanatory power by interpretation for the two premises that include a conditional is as follows: Mental Model Theory interpretation explains 57.2\% of responses (equivalent to the conjunctive interpretation), the biconditional interpretation accounts for $26.2 \%$, and the logic norm for $8.4 \%$. For the test premises the cumulative marks for each norm are $54.7 \%$ for Mental Models, and 9\% on the logic norm; the Mental Model Theory forecast outperforms the other norms. The majority $(61.1 \%)$ of responses to control premises fall on the predicted value, a high score given the difficulty of the problems. Questions using the problem item p(at least A) produce answers spread over several clusters. In the original experiment, about one in four 
respondents hit the logical norm with premise $\mathrm{C} 1 A$ and $(B$ xor $C)$ and problem $p($ at least $A)$; we observe one in five responses on the logic norm. About one in three participants estimated $p($ at least $A)$ to be zero, which can be explained by an interpretation of the item as $p$ (only $A)$. One out of four participants estimates $p$ (at least $A$ ) to be $50 \%$, compared to $40 \%$ of respondents in the original experiment by Johnson-Laird et al. (1999, p. 75), who view this as a pragmatic reinterpretation of the premise. The number of items with an at least formulation is equal for both the test and control conditions.

Linear Model for Experiment 2: Akin to the linear model applied in Experiment 1, we take the complexity of mental representations as the theoretically most relevant predictor of performance. The type of premise is used as a predictor to reflect this complexity. We further include as covariates the item-specific differences and the mode of entry. The dependent variable is the accuracy of responses according to the logical norm and the propensity to answer according to the Mental Model Theory forecast where there exists a bias, respectively. As in Experiment 1 , gender is not a significant predictor. Separate models are run for the test and the control condition. The type of premise appears as a significant predictor of performance in the test condition, for both the logical norm and the Mental Model Theory forecast ${ }^{4}$. In the test group, we find the impact of the entry mode on logical answers to be small $(d=-0.115)$ and not significant $(p>0.2)$, whereas entry the mode has a significant and medium effect $(d=0.345$, $p<0.00022)$ on the propensity to answer as forecasted by the Mental Model Theory. In the control group, none of the predictors of interest (entry mode or premise) is significant. Both the linear model and the logistic regression lead to equivalent conclusions as far as significance is concerned.

From the model of the control condition, we note that problem items like $p$ (at least $A$ ) and $p($ at least $B$ ) are estimated as being more difficult than the others, with odds of answering correctly decreasing by $91 \%\left(z=-4.794, p<1.7 * 10^{-6}\right)$ compared to the item $p(A$ and $B)$. The difficulty of premises according to the linear model is more uniform in the control condition than in the test condition. The test premise E2. A or, If B then $C$, but not all three appears to be the most difficult one in terms of logical reasoning, with a strong bias in favour of the Mental Model Theory forecast. These linear models explain part of the variance observed $\left(R^{2}=0.05\right.$ for logical interpretation; $R^{2}=0.23$ for the Mental Model Theory forecast as the dependent variable). Other factors that go beyond the scope of this paper also have an impact.

\section{General Discussion}

Scope of Application of the Mental Model Theory: Does extensional reasoning apply to estimating probabilities? The explanatory power of the Mental Model Theory reaches 67.2\% when considering the four different interpretations (logical, mental model theoretical, conjunc-

\footnotetext{
${ }^{4}$ In the analysis of variance, the type of premise factor has an F-value $=4.741$ with $p<0.01$ for the logical norm and an $\mathrm{F}$-value $=50.57$ with $p<2.2 * 10^{-16}$ for the Mental Model Theory forecast. We use factorial ANOVA to get the contribution to the explained variance of each effect.
} 
tion, biconditional) for both experiments. Any response that falls on a value forecasted by one of these four interpretations is evidence for the application of extensional reasoning to models using the equiprobability and proportionality principles. The majority of responses are explained by a combination of equiprobability and proportionality principles. The equiprobability and proportionality principles are the most versatile components of the Mental Model Theory of Extensional Reasoning because they may be applied to a wide range of initially built-up mental representations.

Yet, when the problem item contradicts the premise, a conflict detection and resolution strategy can also explain the results. This could be the case in $24.25 \%$ of observed responses where the contradiction is answered correctly ( $83.1 \%$ of responses to contradictions are correct, which is significantly higher than the average). We conclude that extensional reasoning can explain at least $43 \%$ and up to two-thirds of our results.

Bounded Rationality: The tendency to underestimate the number of mental representations is illustrated by the response pattern of Experiment 2. In cases where the problems presented allow for a bias between the Mental Model Theory forecast and the logical norm, respondents tend to answer along the value predicted by the Mental Model Theory, which is derived from a reduced number of mental models. Relative to the number in expected responses on the norm of one-third (implying three mental models), we observe a 55\% shortfall in actual responses. This shortfall is explained by responses derived from mental representations with fewer models: One quarter of responses at 0.5 should instead be at one-third (or $22.5 \%$ of the answers for which the logic norm is one-third ended up being 0.5), all of these are cases where one mental model is omitted. The Mental Model Theory forecasts the majority $(61.5 \%)$ of these shifted responses. This supports the theory of a parsimonious build-up of mental models, and is consistent with the hypothesis that people underestimate the number of instances and build an incomplete mental representation, exemplifying bounded rationality.

The hypotheses of the parsimonious build-up and the principles of equiprobability and proportionality are applicable to the majority of responses that correspond to an interpretation (material implication, mental model theoretical, conjunction and biconditional). When these interpretations yield different forecasted answers, most responses fall on the predictions based on a simplified mental representation. Results for the premises that include a conditional support the assumption that the implicit model of the conditional is dropped when the premise includes a disjunction. The truth principle of the Mental Model Theory, postulating that only true possibilities are represented, is the primary explanation for the parsimonious build-up of mental representations. A parsimonious build-up of models occurs in $19.3 \%$ of all responses: $61.1 \%$ of questions posed with a bias are answered with evidence of a parsimonious build-up.

How much can the number of mental representations explain? The performance scale from easier premises (conjunction and exclusive disjunctions) to more difficult premises (disjunction, conditional) estimated by linear modelling confirms the prediction of the Mental Model Theory that the more mental models a reasoner has to process, the harder the task is (Johnson-Laird \& Byrne, 1991). The number of models determines the extension, which, in 
turn, governs the computation of the probability. Given a specific number of mental models, the mix of models is shaped by the interpretation: When the interpretations produce three different forecasts, a wide variation in estimated probabilities follows. The type of mental representation driven by the interpretation and the number of its models determine the response. The four interpretations of each (conditional) premise account for most of the clusters of answers that we observe. Applying the Mental Model Theory of Extensional Reasoning to representations built according to the logical, mental model theoretical and biconditional interpretations of the conditional, accounts for $66.2 \%$ of responses in the test condition and $63.8 \%$ of all responses in Experiment 2, and up to $69.8 \%$ in Experiment 1. The Mental Model Theory has a higher explanatory power than logical-rule based predictions. Besides the simplified representation illustrating bounded rationality, the use of heuristics (mental short-cuts) by respondents to solve problems can lead to further departures from norms. This may explain part of the responses not accounted for by the Mental Model Theory.

Evidence for the Principles of Equiprobability and Proportionality: In both experiments combined, the mental model theoretical and logical interpretations explain $64.2 \%$ of responses and the mental model theoretical, logical and biconditional interpretations explain $65.4 \%$; the conjunction interpretation of the conditional is not included in this count because it relies on one mental model only. We conclude that the principles of equiprobability and proportionality are applicable to the majority of responses.

Robust Difference between Logical and Alternative Interpretations: The difference in accuracy estimates in Experiment 2 between the mental model theoretical (lower bound of $95 \% \mathrm{CI}$ graphical entry $46.8 \%$, to upper bound of $95 \%$ CI numerical entry $67.0 \%)$ and logic $(28.5 \%$ to $37.9 \%$ ) interpretations remains significant after considering the variability introduced by the use of two different entry modes. The effect of using the more narrow $\pm 4 \%$ band in coding (instead of $\pm 5 \%$ ) the answers is very small: In Experiment 1, two out of 1143 answers end up classified as erroneous rather than falling on an interpretation's norm, a drop of 0.17 percentage points in the explanatory power of the logical, mental model theoretical and conjunction interpretations combined. In Experiment 2, an additional four out of 898 answers are classified as erroneous, a drop of 0.45 percentage points in the explanatory power. Hence we consider our analysis and findings to be robust. While running this experiment, we noticed that the vast majority of participants mastered the task at hand without requiring any assistance. We conclude that the abstract and complex formulations are suited to people who possess a good educational background, like our sample of university students. The significant difference between the number of logical responses and the alternative interpretations substantiates the hypothesis of people's bounded rationality.

Comparison to the Original Experiment: In our larger sample of responses, we note relatively fewer responses predicted by either logical rules or the Mental Model Theory. In particular, we note a significant $\left(\chi^{2}(1)=10.29, p=0.0013\right)$ drop in answers predicted by the Mental Model Theory with a small to medium effect size $(w=0.1741)$ with the conditional 
premise; for the inclusive disjunction, the effect is small $(w=0.1142)$ and barely significant $\left(\chi^{2}(1)=3.6361, p=0.0565\right)$. For premises with three objects, the effect size $(w=0.0521)$ for the decrease in responses on either norm is small. In the case of the test premise E1 A or both $B$ and $C$, not all three, our results corroborate the bias towards estimates based on reduced mental models and approximate the original figures.

Method of Eliciting Probability Estimates: We review the effect of the entry mode and the no-clue field on the number of no-clues, incidence of 50\% answers, accuracy of responses, and usage of scale, and speculate about the mitigation of guessed-responses.

We observe a small rate of no-clue answers in $1.8 \%$ of the 2078 responses. There is a higher propensity to respond in the graphical entry mode where only $0.6 \%$ of the responses are noclues, whereas $2.7 \%$ of the respondents in the numerical entry mode use the no-clue field, which is a small effect $\left(\chi^{2}(1)=12.57, p=0.00039, w=0.0777\right)$. An increase in the usage of the graphical entry mode is also reported by Fischhoff and Bruine de Bruin (1999, p. 152), where the 'absolutely no idea' field was used almost twice as frequently in numerical entry at $13.9 \%$ compared to $7.9 \%$ in graphical entry $\left(\chi^{2}(1)=4.476, p=0.0344, w=0.096\right)$. Thus, we observe a similar increase in the use of the no-clue field in the numerical entry mode compared to graphical entry.

A drop in usage of the $50 \%$ response when responding on a graphical scale, compared to numerical entry, as reported by Fischhoff and Bruine de Bruin (1999, p.153; reported $\chi^{2}(1)=5.92$; implied effect size 0.117 , power $=0.68$ ), is not observed in our study: Our effect size is small $\left(w=0.0177, \chi^{2}(1)=0.5868, p=0.4437\right)$ and not significant. This small effect is not consistent amongst various problems. We conclude that guesses are more effectively captured in the numerical entry mode by the no-clue field because of the larger incidence of no-clues in the numerical entry mode and the lack of difference in the use of the middle of the scale.

In Experiment 1, we noted that the accuracy of responses in the numerical entry mode was somewhat higher than the accuracy in the graphical entry mode. In Experiment 2, responses from the control group show no any significant difference, whereas a small but significant difference in accuracy is forecasted for the test group. The difference in accuracy between the numerical and graphical entry modes is only partially accounted for by the small effects of usage of scale and use of the no-clue field.

When comparing all answers across both experiments, we observe a small to medium ( $w=$ $0.1655)$ effect of the entry mode on the usage of the scale $\left(\chi^{2}\right.$ test: $\chi^{2}(10)=51.13, p=$ $1.65 * 10^{-7}$ ): Participants in the graphical entry condition tended to shy away from the edges of the scale, that is, from the zero and $100 \%$ values. An item by item review reveals that there are no consistent differences in response patterns. The overall difference between the two entry modes is small and statistically significant. Because the difference is small, we pool data from both entry modes for analysing the other topics. We conclude that guesses are more effectively dealt with in the numerical entry mode. 


\section{Conclusion}

The Mental Model Theory that accounts for the parsimonious build-up of mental representations, has a good explanatory power. The parsimonious build-up of mental models leads to available alternatives being overlooked. Reasoning based on simplified mental models produces certain biases, particularly a deviation from purely logical responses built on complete mental representations. Akin to decision makers who overlook some of the alternatives, reasoners have a leaning to build simplified mental representations, and both act with bounded rationality. The parsimonious build-up of mental models is explained by the truth principle and by alternative interpretations that simplify the premises. Our results are compatible with two strategies (extensional reasoning or conflict detection) being used to process mental representations.

The elicitation of probability estimates with two different entry modes (numerical or graphical) shows that a small but significant difference exists in terms of making use of the extreme values of the scale. The better accuracy of responses with a numerical entry mode comes in combination with a more frequent use of the no-clue entry field.

Opportunities for further research include the investigation of individual differences and the level of expertise in statistical reasoning as additional covariates to explain the quality of responses. Assessing the level of expertise could let us delimit 'naïve' individuals from people with statistical and combinatorial reasoning competence. A probe of the tagging of mental models hypothesis can be accomplished by testing participants' ability to solve calculus problems with proportions. Further investigation of response times and possibly of neural correlates via fMRI could be informative regarding which reasoning strategy is employed, and to determine the exact scope of application of the Mental Model Theory of Extensional Reasoning. 
Figure 2

Histograms for the premises $A$ xor ( $B$ and $C), A$ and ( $B$ xor $C$ ), If $A$ then ( $B$ xor $C),(A$ xor $B)$ and $C$.
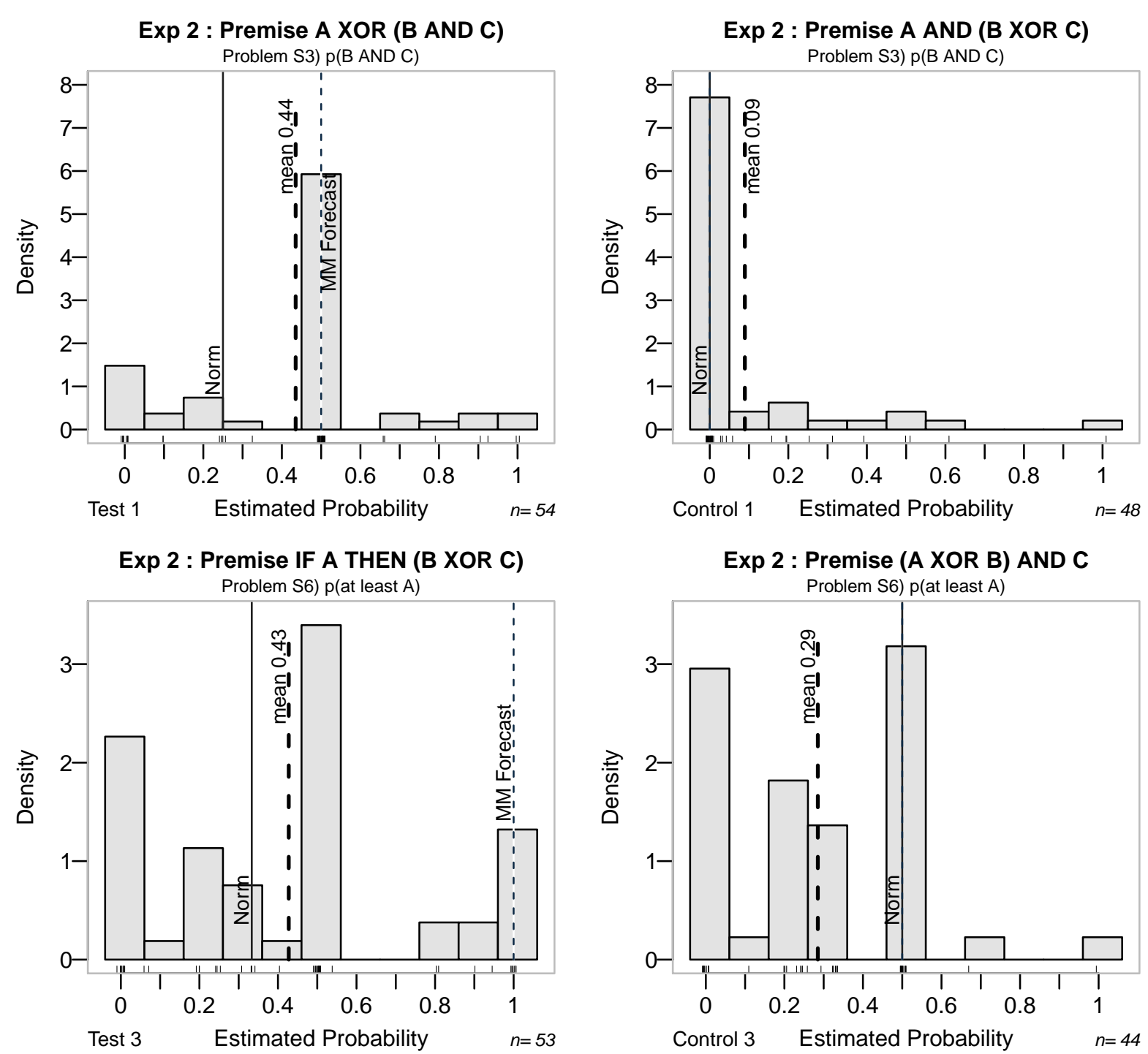

Overlayed norms: Norm= logical norm; MM Forecast= mental model theoretical forecast. Mean= mean of estimated probabilities. 


\section{References}

Barrouillet, P., Gauffroy, C., \& Lecas, J. (2008). Mental models and the suppositional account of conditionals. Psychological Review, 115, 760-772. doi: 10.1037/0033-295X.115.3.760

Byrne, R., \& Johnson-Laird, P. (2009). 'If' and the problems of conditional reasoning. Trends in Cognitive Science, 13, 282-287. doi: 10.1016/j.tics.2009.04.003

Carey, J., \& White, E. (1991). The effects of graphical versus numerical response on the accuracy of graph-based forecasts. Journal of Management, 17, 77-96. doi: $10.1177 / 014920639101700106$

Chevalley, T., \& Schaeken, W. (2013). Is the question clear enough? Learning to understand the premise used in extensional reasoning experiments. Unpublished manuscript, 1-6.

Christensen, A. (2013). Paper beats computer screens. ScienceNordic. Retrieved 14 April 2014, from http: / / sciencenordic.com/paper-beats-computer-screens

Cohen, J. (1988). Statistical power analysis for the behavioral sciences. Hillsdale: Erlbaum.

Evans, J., Handley, S., Neilens, H., \& Over, D. (2007). Thinking about conditionals: A study of individual differences. Memory \& Cognition, 35, 1772-1784. doi: 10.3758/BF03193509

Evans, J., Handley, S., Neilens, H., \& Over, D. (2008). Understanding causal conditionals: a study of individual differences. Quarterly Journal of Experimental Psychology, 61, 1291-1297. doi: 10.1080/17470210802027961

Fischhoff, B., \& Bruine de Bruin, W. (1999). Fifty-fifty $=50 \%$ ? Journal of Behavioral Decision Making, 12, 149-163. doi: 10.1002/(SICI)1099-0771(199906)12:2 149::AID-BDM314 3.0.CO;2-J

Fox, C., \& Rottenstreich, Y. (2003). Partition priming in judgment under uncertainty. Psychological Science, 14, 195-200. doi: 10.1111/1467-9280.02431

Ihaka, R., \& Gentleman, R. (1996). R: A language for data analysis and graphics. Journal of Computational and Graphical Statistics, 5, 299-314. doi: 10.1080/10618600.1996.10474713

Johnson-Laird, P., \& Byrne, R. (1991). Deduction. London / Hove: Laurence Erlbaum.

Johnson-Laird, P., Byrne, R., \& Schaeken, W. (1992). Propositional reasoning by model. Psychological Review, 99, 418-439. doi: 10.1037/0033-295X.99.3.418

Johnson-Laird, P., Legrenzi, P., Girotto, V., Legrenzi, M., \& Caverni, J. (1999). Naive Probability: A Mental Model Theory of Extensional Reasoning. Psychological Review, 106, 62-88. doi: 10.1037/0033-295X.106.1.62

Noyes, J., \& Garland, K. (2008). Computer- vs. paper-based tasks: Are they equivalent? Ergonomics, 51(9), 1352-1375. doi: 10.1080/00140130802170387

Oberauer, K., Geiger, S., Fischer, K., \& Weidenfeld, A. (2007). Two meanings of IF? Individual differences in the interpretation of conditionals. The Quarterly Journal of Experimental Psychology, 60, 790-819. doi: 10.1080/17470210600822449

Ridderinkhof, K., van den Wildenberg, W., Segalowitz, S., \& Carter, C. (2004). Neurocognitive mechanisms of cognitive control: The role of prefrontal cortex in action selection, response inhibition, performance monitoring, and reward-based learning. Brain and Cognition, 56, 129-140. doi: 10.1016/j.bandc.2004.09.016

Simon, H. (1957). Models of man, social and rational. New York: John Wiley and Sons. 
Thompson, V. (2000). The task-specific nature of domain-general reasoning. Cognition, 76, 209-268. doi: 10.1016/S0010-0277(00)00082-2

Tversky, A., \& Kahneman, D. (1983). Extensional versus intuitive reasoning: The conjunction fallacy in probability judgment. Psychological Review, 90, 293-315. doi: 10.1037//0033295X.90.4.293

Wason, P. (1966). Reasoning. In B. Foss (Ed.), New horizons in psychology (pp. 135-151). Harmondsworth Middlesex England: Penguin Books. 


\section{Appendix A: Mental Models for Experiment 2}

Table 8

Initial Mental Models and Fully Explicit Models for Premises in Experiment 2

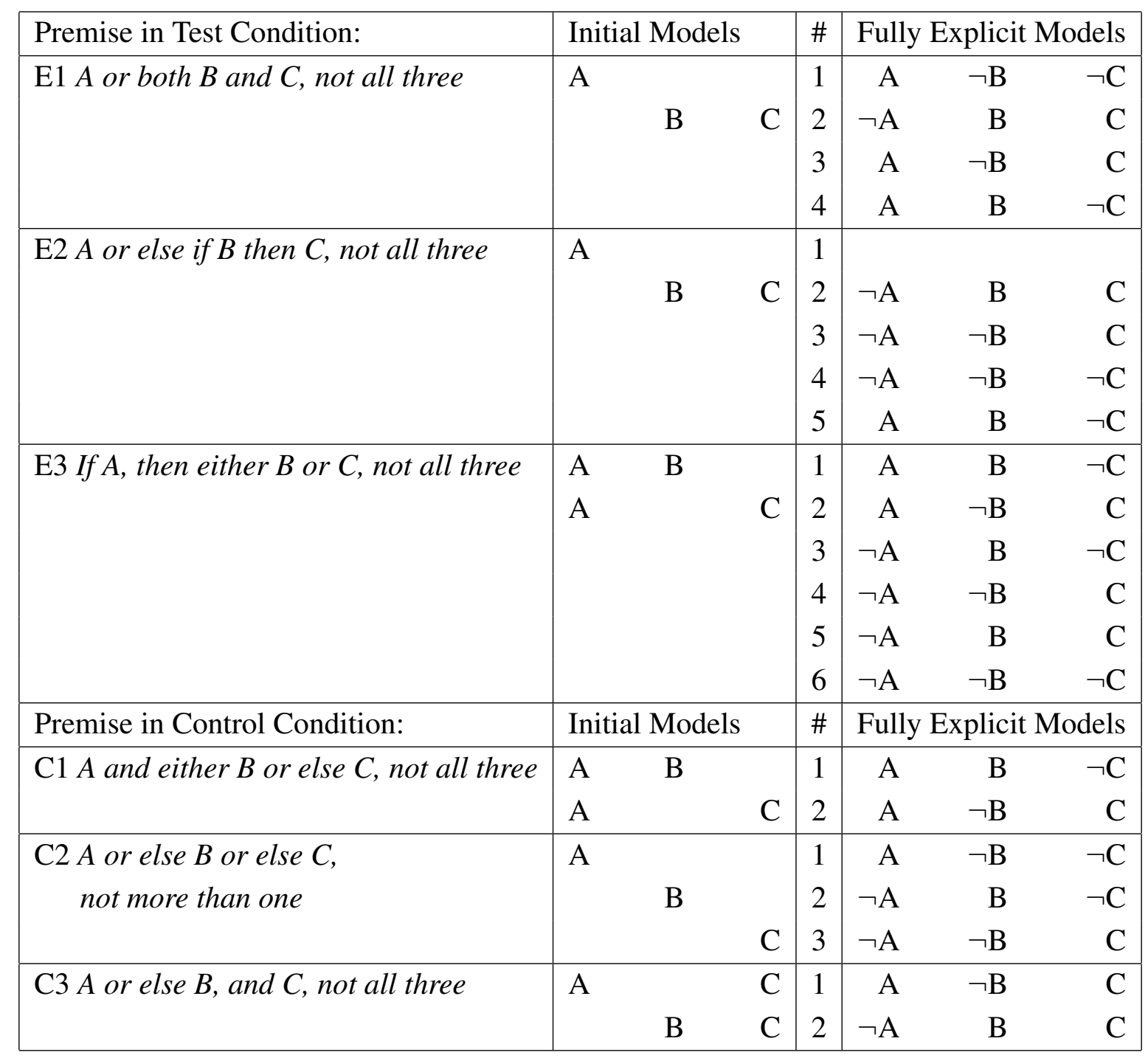

Note: Models for biconditional interpretation of premise E2: \#1,2,4. Models for biconditional interpretation of premise E3: \#1,2,5,6. The conjunction interpretation generates the same initial models as the mental model theoretical interpretation. In the control condition, the initial mental models lead to the same predicted values as the fully explicit models. $\neg$ is a tag representing negation. 